\title{
Estado de la cuestión de los centros y programas de escritura en Latinoamérica
}

State of the Art of Writing Centers and Programs in Latin America

Estado da arte das oficinas e programas de redação na América Latina

\author{
Violeta Molina-Natera** (iD hetps;//orcid.org/0000-0002-6636-7222 \\ Karen Shirley López-Gil ${ }^{* * *}$ (iD) htrps://orcid.org/0000-0001-9826-0799
}

Doi: 10.17227/rce.num78-8066

Para citar este artículo: Molina, V. y López, K. (2020). Estado de la cuestión de los centros y programas de escritura en Latinoamérica. Revista Colombiana de Educación, 78, 97-119. http://doi.org/10.17227/rce.num78-8066

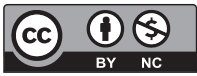

* En este artículo se presentan resultados parciales de un proyecto de investigación que se adelantó en la Pontificia Universidad Javeriana, seccional Cali (Colombia), de enero de 2015 a enero de 2016 y contó con la financiación de dicha institución bajo el Registro de Investigación 00004081 de la Convocatoria Capital Semilla.

** Doctora en Educación. Profesora investigadora del Departamento de Comunicación y Lenguaje, Facultad de Humanidades y Ciencias Sociales. Investigadora del Grupo Procesos y Medios de Comunicación, línea de Estudios del Lenguaje, Pontificia Universidad Javeriana. Correo electrónico: vmolina@javerianacali.edu.co

*** Doctora en Educación. Profesora investigadora del Departamento de Comunicación y Lenguaje, Facultad de Humanidades y Ciencias Sociales. Directora del Centro de Escritura Javeriano. Investigadora del Grupo Procesos y Medios de Comunicación, línea de Estudios del Lenguaje. en Pontificia Universidad Javeriana. Correo electrónico: karens(ajaverianacali.edu.co 


\section{Resumen}

Esta investigación buscaba identificar las teorías, prácticas y procesos de gestión de los centros y programas de escritura en universidades de América Latina. Para ello, se propuso un estudio exploratorio que tuvo como principal instrumento un cuestionario en línea, basado en dos investigaciones norteamericanas: "The Writing Center Research Project" y "The Writing Program Administration Census Project". Participaron en la investigación 23 directores de centros y programas de escritura de la región. Los resultados que se presentan aquí, a partir de un análisis predominantemente cuantitativo, evidencian una variedad de programas muy jóvenes en su conformación. La escritura empieza a incluirse en las agendas programáticas de las universidades latinoamericanas, teniendo en cuenta el respaldo institucional conferido a estas iniciativas en los últimos diez años. Respecto a los fundamentos teóricos, se encontró una diversidad de enfoques y posturas que configuran estos centros y programas de forma distinta a sus antecesores en Norteamérica. Los programas incorporan prácticas que tienen que ver, en su mayoría con el acompañamiento individual a través de tutorías o con el trabajo colaborativo entre docentes de lengua y docentes de otras disciplinas. La gestión administrativa plantea un desafio especial en cuanto a la medición del impacto de los programas. Los directores asumen una serie de funciones que requieren de una capacitación integral en tareas administrativas, con la cual no se cuenta aún en la región. Estos resultados evidencian la necesidad de fortalecer los procesos de gestión de estas iniciativas y la pertinencia de consolidar un campo propio de investigación.

\section{Palabras clave}

educación superior; escritura; programas; calidad educativa; talleres de escritura; mejoramiento de la escritura

\section{Keywords}

higher education; writing; programs; education quality; writing workshops centers; writing improvement program

\begin{abstract}
This research aimed to identify the theories, practices and management processes of writing centers and programs in Latin American universities. To that end, we proposed an exploratory study, whose main instrument was an online survey based on two North American researches: "The Writing Center Research Project" and "The Writing Program Administration Census Project." Twenty-three directors of writing centers and writing programs in the region participated in the research. The results presented in this paper from a predominantly quantitative analysis, showed a variety of very young programs in their conformation. Writing begins to be included in the program agendas of Latin American universities, taking into account the institutional support given to these initiatives over the last 10 years. As regards the theoretical foundations, a diversity of approaches and theoretical positions were found that configure these centers and programs in a different way to their predecessors in North America. The programs incorporate practices that have to do, mostly, with individual accompaniment through tutorials or with the collaborative work between language teachers and teachers from other disciplines. Administrative management represents a special challenge in measuring the impact of programs. Directors assume a series of functions that require an integral training in administrative tasks that is not found in the region. These results demonstrate the need to strengthen the administrative processes of these initiatives and the relevance of consolidating their own research field
\end{abstract}

\section{Resumo}

Esta pesquisa buscou identificar as teorias, práticas e processos gerenciais das oficinas e programas de redação nas universidades latino-americanas. Para tanto, propôs-se um estudo exploratório que utilizou como instrumento principal um questionário online, baseado em duas pesquisas norte-americanas: "The Writing Center Research Project" e "The Writing Program Administration Census Project". 23 diretores de centros de escrita e programas da região participaram da pesquisa. Os resultados apresentados aqui, baseados em uma análise predominantemente quantitativa, mostram uma variedade de programas muito novos em sua conformação. A escrita começa a ser incluída nas agendas programáticas das universidades latino-americanas, levando em consideração o apoio institucional dado a essas iniciativas nos últimos dez anos. Em relação aos fundamentos teóricos, encontramos uma diversidade de abordagens e posições que configuram essas oficinas e programas de forma diferente de seus antecessores na América do Norte. Os programas incorporam práticas que têm a ver, na maior parte, com acompanhamento individual através de tutoriais ou com trabalho colaborativo entre professores de línguas e professores de outras disciplinas. A gestão administrativa representa um desafio especial em termos de medir o impacto dos programas. Os diretores assumem uma série de funções que exigem treinamento abrangente em tarefas administrativas, que ainda não estão disponíveis na região. Esses resultados mostram a necessidade de fortalecer os processos de gestão dessas iniciativas e a relevância de consolidar um campo de pesquisa adequado.

\section{Palavras-chave}

ensino superior; redação; programas; qualidade educacional; oficinas de redação; aperfeiçoamento de programas de redação 


\section{Introducción}

La lectura y la escritura en las universidades se han convertido en indicadores de la calidad académica en Latinoamérica, y en el mundo, en los últimos años. Sin embargo, distintos estudios diagnósticos y pruebas estatales han Ilamado la atención sobre las deficiencias de los estudiantes tanto en la comprensión como en la producción de textos (Carlino, 2005; González, 2010; Backhoff, Velasco y Peón, 2013; Pérez y Rincón, 2013) y la relación entre estos problemas y la deserción académica (Olave, Rojas y Cisneros, 2013). Por esta razón, las diferentes instancias universitarias se han cuestionado y se han planteado alternativas que faciliten su desarrollo, con enfoques diversos, respondiendo a propósitos distintos y con abordajes orientados a la población estudiantil o al cuerpo profesoral.

Dentro de estas iniciativas, además de los cursos introductorios de lectura y escritura, se encuentran los programas de índole institucional que se ocupan de estos procesos a través de diferentes prácticas. Por un lado, están los centros de escritura, que a través de tutorías personalizadas proponen estrategias para que los tutorados desarrollen habilidades de escritura de textos académicos (Harris, 1988). Estas tutorías pueden ser presenciales o virtuales, y son ofrecidas por tutores pares académicos, profesores o estudiantes de niveles superiores (Gillespie y Lerner, 2004). Por otro, están los programas de escritura, que pueden tener una variedad de enfoques que van desde cursos iniciales o especializados de escritura, hasta programas de formación docente para desarrollar prácticas de lectura y escritura en las asignaturas o programas con enfoque de escritura a través del currículo (McLeod, 2007). El común denominador de estas iniciativas es que cuentan con respaldo institucional para atender a gran parte o la totalidad de la población estudiantil o profesoral.

En algunos casos surgen en respuesta ante un déficit que se establece en los estudiantes, quienes no leen ni escriben y se tiende a buscar estrategias que ayuden a superar esa deficiencia. Hay otros casos en los que la intención no es necesariamente superar las deficiencias, sino ayudar a desarrollar una serie de habilidades con el fin de acompañar los procesos de aprendizaje universitario (por ejemplo, Moyano y Natale, 2012; Santos, 2016). De ahí que hablemos de una variedad de programas con particularidades que los hacen únicos y con propuestas de trabajo que obedecen a las necesidades y a las realidades de cada institución. Tenemos, por una parte, los centros de escritura inspirados en los writing centers norteamericanos y cuyo eje central son las tutorías personalizadas de escritura (Molina-Natera, 2014) (aunque también se incluyen tópicos como lectura académica, oralidad, competencia en el manejo de información, etc.). Por otra parte, están los programas de escritura, que pueden definirse como un conjunto de actividades articuladas que usan una serie

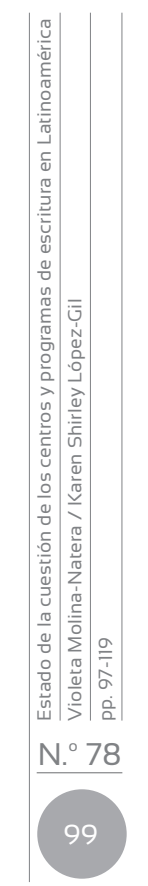


de recursos para lograr resultados específicos y documentables. Aunque tienen orientaciones diferentes, en su mayoría su enfoque se centra en el trabajo en las asignaturas, bien sea las asignaturas de lengua que se ofrecen al inicio de las carreras, o bien los cursos disciplinares en los que los docentes de lengua apoyan el trabajo de los disciplinares, entre otras posibilidades (White, Elliot y Peckham, 2015).

En Latinoamérica la aparición de los centros y programas de escritura se viene dando a partir de los últimos diez años y cobra una relevancia significativa debido al impacto que pueden tener en las comunidades académicas. No obstante, el trabajo que realizan estos programas es un objeto de investigación inexplorado hasta el momento en este contexto y la mayoría de las instituciones desconocen el trabajo que los demás vienen adelantando (Molina-Natera, 2015). Además, las personas que lideran estas iniciativas no disponen de una oferta formativa para un trabajo tan complejo y a la vez específico, debido a que en esta región no hay programas que formen para esta actividad y no se reconoce aún el cargo de director de programa de escritura con todas las demandas que implica. Para dar respuesta a dicho problema, se propuso un proyecto de investigación que tenía como objetivo caracterizar los fundamentos conceptuales, las estrategias de abordaje y los procesos de gestión académica que siguen los programas y centros de escritura de Latinoamérica. En este artículo se presentan los resultados de las preguntas de la encuesta, desde un análisis predominantemente cuantitativo.

\section{Método}

\section{Objetivo}

Esta investigación buscaba identificar las teorías, prácticas y procesos de gestión de los centros y programas de escritura en universidades de América Latina. Se planteó un estudio exploratorio (McMillan y Schumacher, 2005), debido al reciente interés que hay en Latinoamérica por estudiar las características y los impactos de los centros y programas de escritura, y a la escasez de investigaciones y publicaciones al respecto en este contexto.

\section{Instrumento}

En la recolección de información se usó una encuesta en línea (García-Córdoba, 2002; Páramo y Arango, 2008), elaborada a partir de los instrumentos empleados en dos estudios realizados en Estados Unidos: "The Writing Center Research Project"1 y "The Writing Program Administration Census

1 http://writingcenters.org/resources/wcrp/ 
Project" $^{\prime 2}$. El primer proyecto, desarrollado por la International Writing Centers Association (IWCA, 2010), tenía como propósito caracterizar los centros de escritura en Estados Unidos y en otros países. El segundo proyecto, liderado por Gladstein y Fralix (2014), busca establecer un censo de los programas de escritura de Estados Unidos, incluyendo también centros, en instituciones de educación superior de dos y cuatro años. Se contó con las autorizaciones de los coordinadores de estos proyectos para usar los instrumentos y hacer los ajustes pertinentes.

Para aplicar las preguntas de los dos instrumentos en este estudio, se hicieron las adaptaciones correspondientes al contexto social y político de las instituciones de educación superior en Latinoamérica. Los aspectos retomados de The Writing Center Research Project conforman la mayoría de las preguntas de la encuesta, en especial las que tienen que ver con las prácticas de los centros. Los ajustes realizados a este instrumento buscaban ampliar la cobertura de las preguntas a los programas de escritura y no restringirse únicamente a información sobre centros. Por su parte, de "The Writing Program Administration Census Project" se retomaron preguntas principalmente para la categoría de administración. Las preguntas que indagaban sobre las teorías que subyacen a los programas fueron diseñadas por las investigadoras de este estudio.

La encuesta se formuló teniendo en cuenta que no todas las iniciativas usan los mismos criterios para su denominación (centro o programa) y tratando de que las preguntas cubrieran todos los aspectos de su quehacer. El objetivo de la encuesta era obtener información sobre las razones que motivaron el surgimiento de los centros y programas, sus referentes teóricos, las prácticas que implementan, características del programa y de las personas que trabajan en él, así como los procesos de gestión administrativa que sirven de base para su funcionamiento. El instrumento se organizó en 12 secciones, con un total de 118 preguntas, en su mayoría con respuestas de opción múltiple. El instrumento fue validado por dos directores de programas de escritura, quienes evaluaron la coherencia, pertinencia y claridad en la presentación de las preguntas y sugirieron algunos ajustes.

\section{Participantes}

A través del correo electrónico, se invitó a los directores de los 40 centros y programas de escritura, identificados hasta 2015, a responder la encuesta y se contó con 23 participantes. Estos directores podían pertenecer o no a la Red Latinoamericana de Centros y Programas de Escritura (RLCPE). De forma anticipada se envió una versión imprimible en formato PDF que se

2 http://writingcensus.swarthmore.edu/ 
podía revisar para conocer y conseguir la información que se solicitaba. Se incluyó, en primer lugar, el consentimiento informado que daba paso a las preguntas del cuestionario si las personas aceptaban participar en el estudio. Dada la complejidad y la extensión de la encuesta, se programó en la herramienta Lime Survey, de manera que los participantes pudieran acceder a ella y guardar información para responderla en diferentes momentos. Asimismo, se solicitó que adjuntaran documentos en formato textual (actas de constitución, lineamientos institucionales, etc.) y gráfico (fotografías de los espacios) para complementar el análisis (Banks, 2010; Rapley, 2014).

\section{Procedimiento de análisis de datos}

Para el tratamiento de las respuestas cerradas de la encuesta se usaron gráficos de frecuencia y estadísticos de contraste a través de pruebas no paramétricas como Kruskal-Wallis, y prueba de la mediana, debido al tamaño de la muestra (23 participantes) mediante el software SPSS v.22. Para las respuestas abiertas y los documentos en formato textual y gráfico enviados por los participantes se usó la técnica de análisis de contenido cuantitativo (Bardin, 1996, López-Noguero, 2002) con apoyo del software Atlas.Ti 7.0.

\section{Resultados}

Considerando la extensión del cuestionario, se presentan a continuación los resultados más relevantes para las tres categorías propuestas: fundamentación teórica, prácticas y procesos de gestión administrativa, además de la información general de los programas y de sus antecedentes de creación.

El cuestionario fue respondido por 23 de los 40 programas de escritura identificados en el 2015, la mayoría miembros de la RLCPE, según se explica en el anexo 1. Debido a que el cuestionario buscaba abarcar todas las posibilidades de respuesta que involucraran a la variedad de programas existentes, varias de las preguntas no podían ser respondidas por todos. Sin embargo, se observó una significativa carencia de respuestas en preguntas que sí podían responderse por la totalidad de los programas participantes, lo que generó un alto número de datos perdidos.

\section{Información general}

La mayoría de las universidades que respondieron fueron de Colombia (11), seguido de México (5), con representación también de Chile (2), Argentina (2), Uruguay (1), Puerto Rico (1) y República Dominicana (1) (figura 1). Colombia es el país de Latinoamérica que más cuenta con 
centros de escritura, de allí que la mayoría de los directores que respondieron la encuesta provengan de dicho país. En general, participaron universidades, con solo un caso de una institución de educación superior. Correspondían a sedes principales 22 programas y uno a sede regional. De los 23 programas, 16 pertenecían a instituciones educativas privadas y 7 a instituciones públicas.

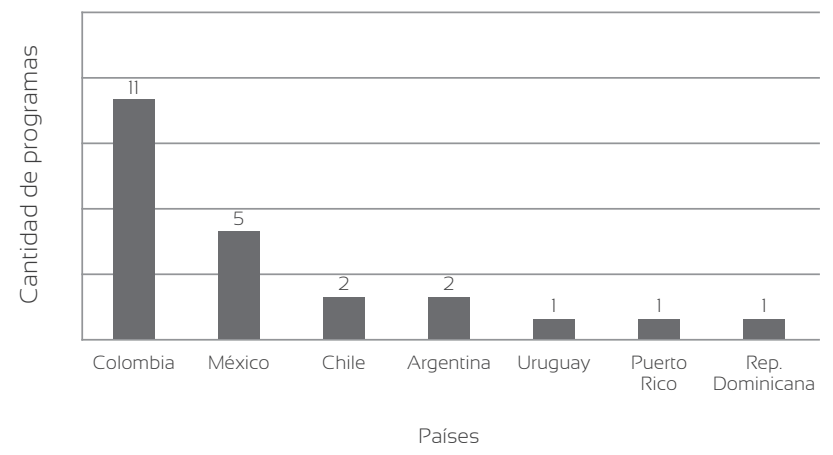

Figura 1.

Distribución de países participantes

Fuente: elaboración propia.

\section{Antecedentes}

El promedio de inicio de los programas es 2009, en un rango entre 1990 y 2015. Sin embargo, puede evidenciarse que el dato 1990 se presenta como un dato atípico y aislado del resto de datos, por lo que la mediana, 2011, representa mejor el tiempo de inicio. Como puede observarse en la figura 2, la mayoría de programas participantes tiene menos de diez años de antigüedad y un alto porcentaje tiene menos de 5 años, lo cual evidencia lo reciente de estos.

En cuanto al tiempo tomado para elaborar el proyecto de creación del programa, en promedio se tomaron 12,5 meses para el diseño de la propuesta. El número menor de meses reportados fue de 3 y el mayor fue 24. En las respuestas al cuestionario se evidencia que 11 proyectos estuvieron liderados por una sola persona, mientras que los otros proyectos fueron propuestos por dos o más. El equipo más grande estuvo conformado por 9 personas.

Respecto a sus perfiles, cinco personas con experiencia en cargos de gestión (dirección de departamento, coordinación de programas académicos) asumieron el liderazgo de los proyectos de creación de programas de escritura y otros tres aparecen acompañando a docentes. En 12 respuestas se presentan a los creadores como docentes de áreas relacionadas con escritura, lingüística, literatura y humanidades. 


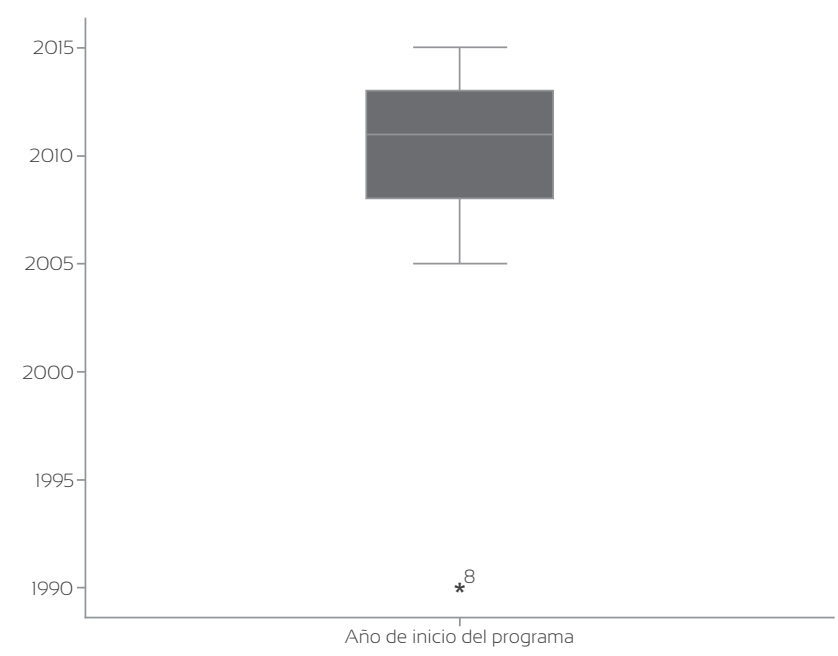

\section{Figura 2}

Año de inicio del programa

Fuente: elaboración propia.

En relación con las razones que motivaron la creación de programas, en el análisis de contenido cuantitativo con el AtlasTi se evidencian 17 respuestas relacionadas con mejorar/fortalecer, aumentar/desarrollar la escritura. Llama la atención la variedad de denominaciones del objeto de interés: habilidades de escritura, competencias de escritura, competencia comunicativa escrita, producción de textos, proceso de escritura y redacción. En cinco respuestas se evidencia un interés por la lectura, además de la escritura. Tres respuestas incluyen como razón de la creación de los programas un interés por mejorar la calidad educativa de la institución y el rendimiento de los estudiantes. Por su parte, una respuesta indica como razón la necesidad de desarrollar el pensamiento crítico, otra respuesta presenta como motivación la innovación tecnológica, mientras que otra se centra en consolidar el quehacer pedagógico.

Una vez iniciados, se observa que los programas tuvieron que enfrentar algunos problemas y la mayoría indicó haber tenido más de una dificultad en este inicio. En la figura 3 se identifica que el principal problema reportado fue el de las representaciones que tenía la comunidad universitaria respecto a la escritura. También se reportaron problemas de apoyo financiero, formación del personal y aspectos locativos. En menor proporción se identificaron problemas relacionados con las políticas institucionales, el tiempo asignado para la gestión y la difusión del programa. 


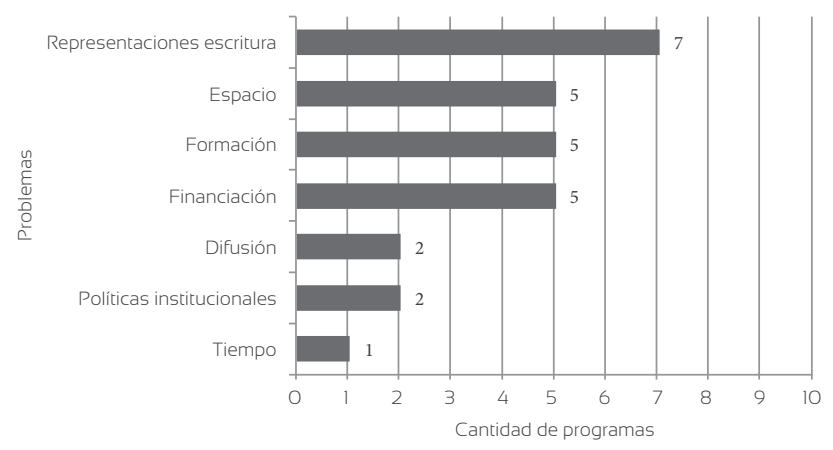

Figura 3

Problemas enfrentados al inicio del programa

Fuente: elaboración propia.

Respecto a las medidas empleadas para enfrentar los problemas iniciales, se identificaron estrategias relacionadas con procesos de formación de profesores y estudiantes/monitores que hacían parte del programa (5 respuestas), como la contratación o asesoría de expertos, la consulta o construcción de materiales de apoyo y las capacitaciones; estrategias relacionadas con procesos de sistematización e investigación para demostrar la pertinencia de la propuesta (4 respuestas); estrategias relacionadas con buscar aceptación por parte de la comunidad académica a través de servicios de acompañamiento a estudiantes y profesores (3 respuestas); estrategias relacionadas con ofrecer el programa como un espacio de práctica formativa o servicio social para vincular a estudiantes, sin que implicara financiación por parte de la institución (2 respuestas); presentación del proyecto ante directivos con poder de decisión (1 respuesta); solicitud a la universidad de apoyo para publicidad del programa (1 respuesta), y persistencia (1 respuesta).

\section{Fundamentación}

En esta categoría se contemplan aspectos relacionados con los fundamentos teóricos y conceptuales en los que se basan los programas participantes. Se encontró que 15 de los 23 programas se basaron en la revisión de otros centros y programas para formular sus propuestas. Solo 6 programas consultaron y recibieron asesorías de pares que trabajaban en programas similares.

En esta categoría se indagó por las fuentes bibliográficas que se usaron como sustento conceptual en las propuestas de creación de los programas, de las cuales se obtuvieron 144 respuestas. A partir de la revisión de los títulos y de los autores, estas referencias fueron clasificadas en siete categorías. Como se observa en la figura 4, 30 de las referencias reportadas 
corresponden a manuales de escritura, 24 a textos sobre escritura en la universidad, 22 a páginas de centros de escritura específicos, 14 a otros textos (sobre TIC, principalmente), 10 a teorías lingüísticas, 6 a educación $y$, aunque 38 referencias corresponden a teorías sobre centros y programas de escritura, cabe resaltar que estas fuentes provinieron únicamente de tres participantes.

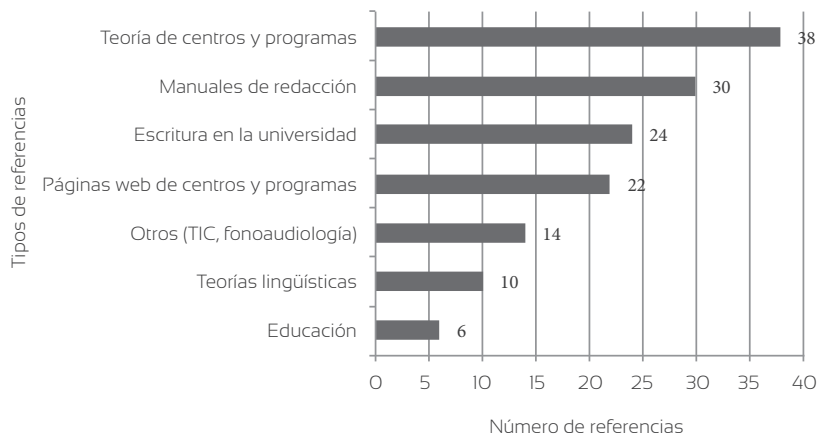

\section{Figura 4}

Fundamentación conceptual para formular el proyecto

Fuente: elaboración propia.

En esta categoría se preguntó también por el acceso a revistas y libros especializados en centros y programas de escritura. De los 23 programas, 14 no cuentan con suscripción a este tipo de revistas, 3 están suscritos a una, y 3, a 2 o más revistas. En cuanto a la disponibilidad de libros sobre centros y programas de escritura, más de la mitad de los programas declararon no tener este tipo de libros disponibles. De los que dicen tener este tipo de libros, un programa no precisa los títulos, 2 se refieren al libro de la autora Paula Carlino (2005) (que, aunque se enfoca en la escritura en la universidad, no es sobre centros y programas de escritura) y las otras 2 respuestas sí especifican textos especializados sobre el tema.

Ahora bien, la fundamentación en estos programas puede provenir del contacto con pares. De los 23 participantes, 13 expresaron que consultan regularmente páginas sobre asociaciones de centros y programas de escritura, las cuales, en su gran mayoría, corresponden a páginas de centros de escritura, pero no de asociaciones que congreguen estos programas. Solo dos respuestas correspondieron a asociaciones existentes y una se refirió a la página de la RLCPE, recién creada. En cuanto a la asistencia a eventos especializados, 13 programas indicaron que habían tenido representación en este tipo de encuentros y 10 especificaron cuáles fueron esos eventos. Finalmente, en esta categoría se observó que el número de publicaciones sobre centros y programas de escritura se reduce a 7 de los 23 participantes, de los cuales solo 5 ofrecieron información sobre dichos documentos. 


\section{Prácticas}

Se pudo observar la diversidad de prácticas que conforman los programas de la región. La mayoría de ellos (11 de 23) son tutorías en centros de escritura, seguido por formación de profesores de otras áreas (escritura a través del currículo). Como se observa en la figura 5, es muy baja la incidencia de estos programas en los posgrados, pues son muy pocos los servicios dirigidos a la escritura a este nivel.

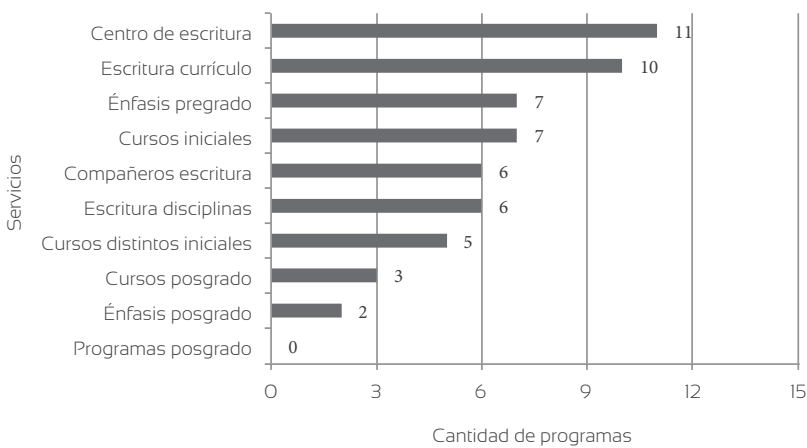

\section{Figura 5}

Servicios que prestan los programas

Fuente: elaboración propia.

El cubrimiento de los programas es amplio, pues 13 de los 23 programas atienden a todos los estudiantes de pregrado, 5 a estudiantes de una carrera o facultad específica, 10 a profesores y personal, y 9 a estudiantes de posgrado, como se muestra en la figura 6.

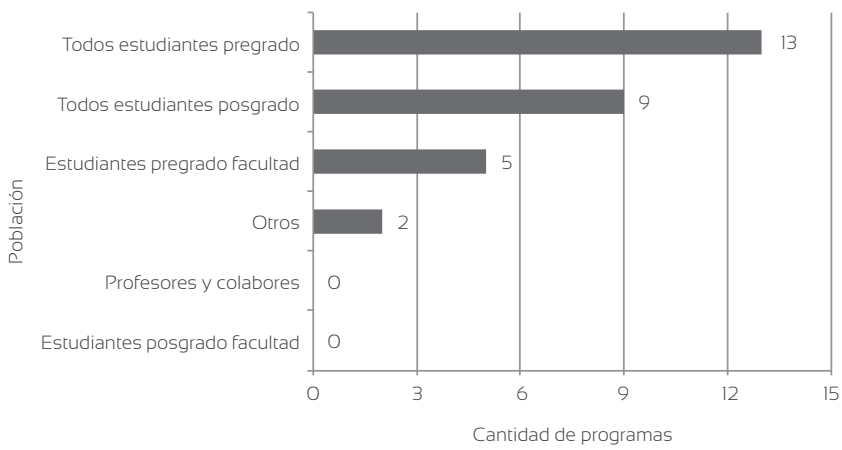

Figura 6

Población atendida en los programas

Fuente: elaboración propia. 
De acuerdo con los datos obtenidos, los programas participantes atienden en promedio 34 horas semanales, aunque esta media tiene una desviación típica alta $(19,34)$. El menor número de horas reportadas fue 4 y el mayor 75. El número de usuarios atendidos también presenta importantes variaciones, con un promedio anual (en 2014) de 326 usuarios. El boxplot (figura 7) evidencia una distribución irregular y asimétrica de la muestra y el rango, cuyo valor mínimo es 0 y máximo 889, también da cuenta de estas importantes diferencias entre los programas.

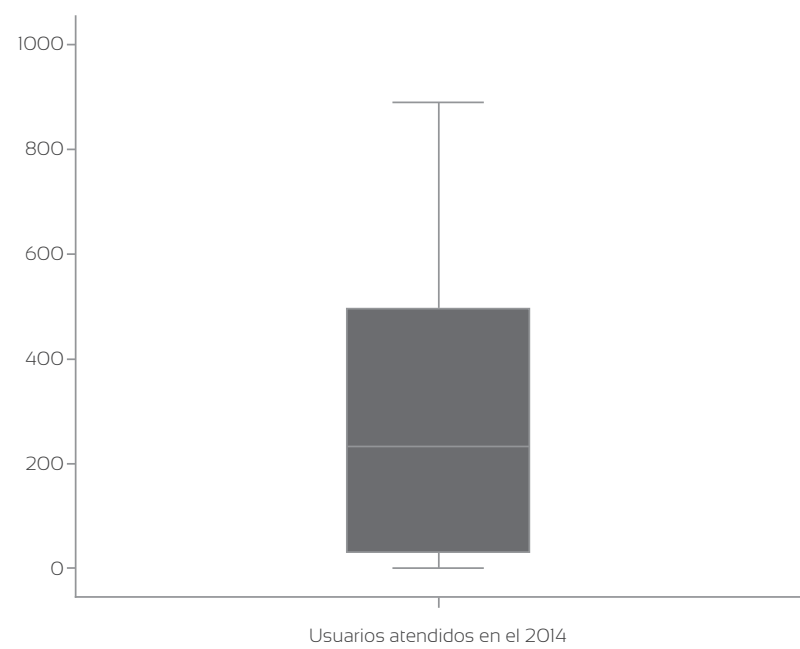

Figura 7

Cantidad de usuarios atendidos en 2014

Fuente: elaboración propia.

En esta categoría se indagó también por las proyecciones de los programas en internet. Se encontró que 13 programas cuentan con página web, principalmente de tipo informativa (información sobre los servicios, contacto, equipo de trabajo), mientras que 7 programas se promocionan en las redes sociales.

\section{Gestión}

Esta categoría está relacionada con el trabajo de diseño, monitoreo, evaluación, manejo del personal, relaciones institucionales y todas las tareas administrativas que implica un programa de esta naturaleza. Como se observa en la figura 8 , se encontró una variedad de unidades académicas a las cuales están adscritos los programas, pues 6 dependen de la vicerrectoría académica, 5 de departamentos de lenguas/español, 4 de facultades de humanidades/ciencias sociales, 3 de otras instancias y solo 1 es una unidad académica independiente. 


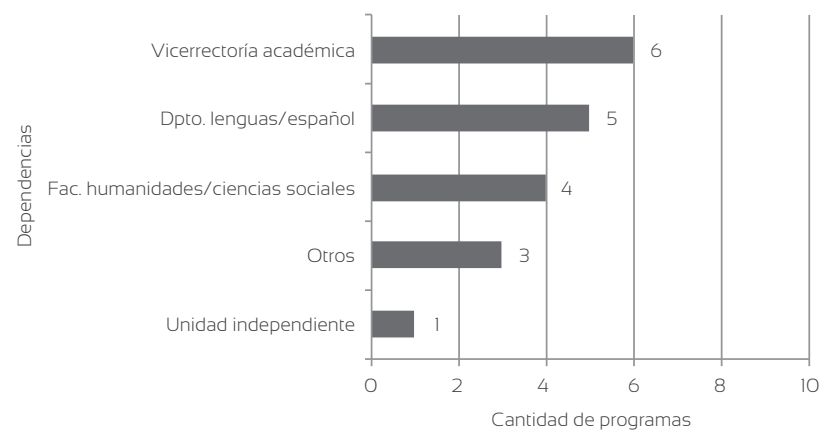

\section{Figura 8}

Unidades de las que dependen los programas

Fuente: elaboración propia.

En cuanto a la ubicación que las instituciones confieren para el funcionamiento de los programas, solo 2 de ellos cuentan con espacios específicos para sus operaciones, mientras que los otros programas funcionan en espacios como la biblioteca, las oficinas de los profesores, internet, salones de clase u otros espacios (figura 9).

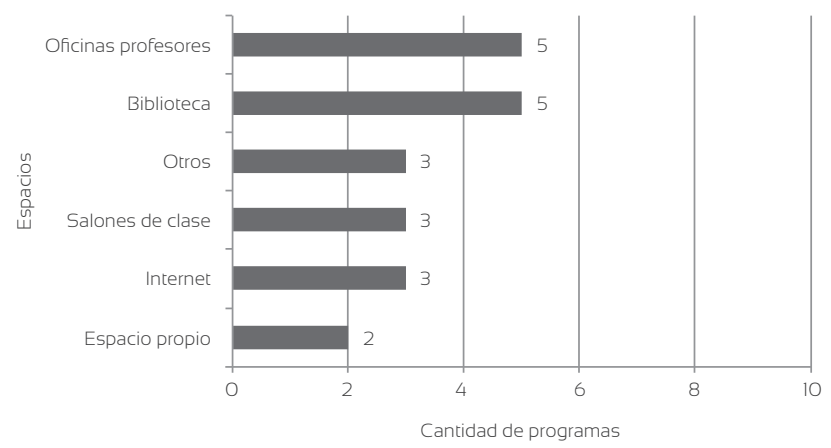

\section{Figura 9}

Espacios en los que funcionan los programas

Fuente: elaboración propia.

Por otro lado, se evidenció que se cuenta con diversidad de cargos en estos programas; en general, los programas tienen un director o coordinador general y dependiendo de sus servicios cuentan con tutores-estudiantes, profesores, coordinadores de área y otros cargos (asistentes técnicos y auxiliares administrativos) (figura 10). 


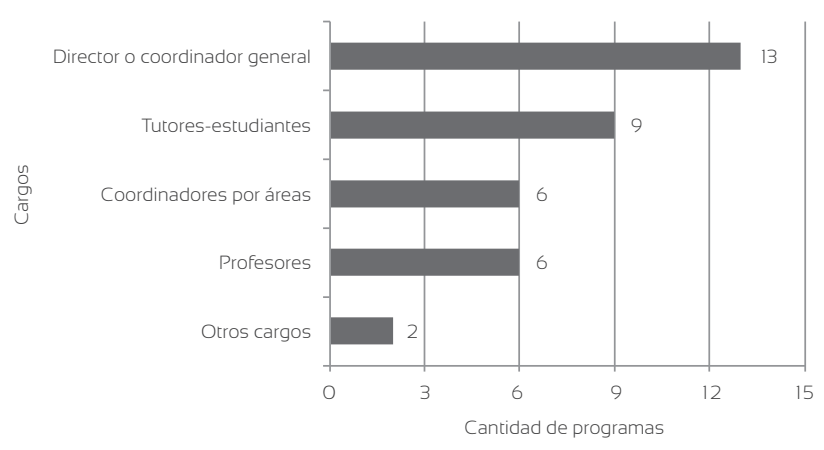

Figura 10

Cargos establecidos en los programas

Fuente: elaboración propia.

La evaluación de los programas de escritura parece ser una actividad que la mayoría de los programas aún no lleva a cabo, pues 14 de los 23 programas manifiesta no hacerlo, 3 sí lo hacen y 3 explican que esto no aplica por ser programas que están iniciando. Asimismo, solo 5 programas registran información sobre la eficacia de sus servicios, 6 indican que no lo hacen y para 9 esto no aplica a sus programas. Por su parte, 12 programas manifiestan elaborar informes periódicos para las unidades de las cuales dependen. Estos datos coinciden con la posibilidad de contar con presupuesto propio, pues solo 5 programas lo tienen.

La persona que tiene a su cargo el manejo de estos programas tiene un perfil diverso. En promedio el tiempo de experiencia del director es de 4 años, con un mínimo de 1 y un máximo de 15. La mayoría cuenta con estudios de maestría (10 casos), 3 son doctores y solo 1 cuenta con especialización como nivel máximo de estudios. Sus funciones abarcan en promedio 12 actividades distintas a cargo, con un mínimo de 5 y un máximo de 25. La figura 11 muestra que las funciones más frecuentes son programar eventos y actividades del programa, hacer investigación y elaborar materiales para el programa.

Por último, el entrenamiento para capacitar al personal que labora en el programa se reporta variado en cuanto a sus estrategias. La mayoría de los procesos formativos se llevan a cabo con los tutores (estudiantes universitarios en rol de monitores o, en algunos casos, profesores de escritura). La estrategia que predomina es la realización de una inducción personalizada a los tutores, que se combina con talleres a lo largo del año. Algunos programas hacen una semana de entrenamiento intensivo antes de empezar el semestre y apenas dos cuentan con un curso semestral completo (figura 12). 


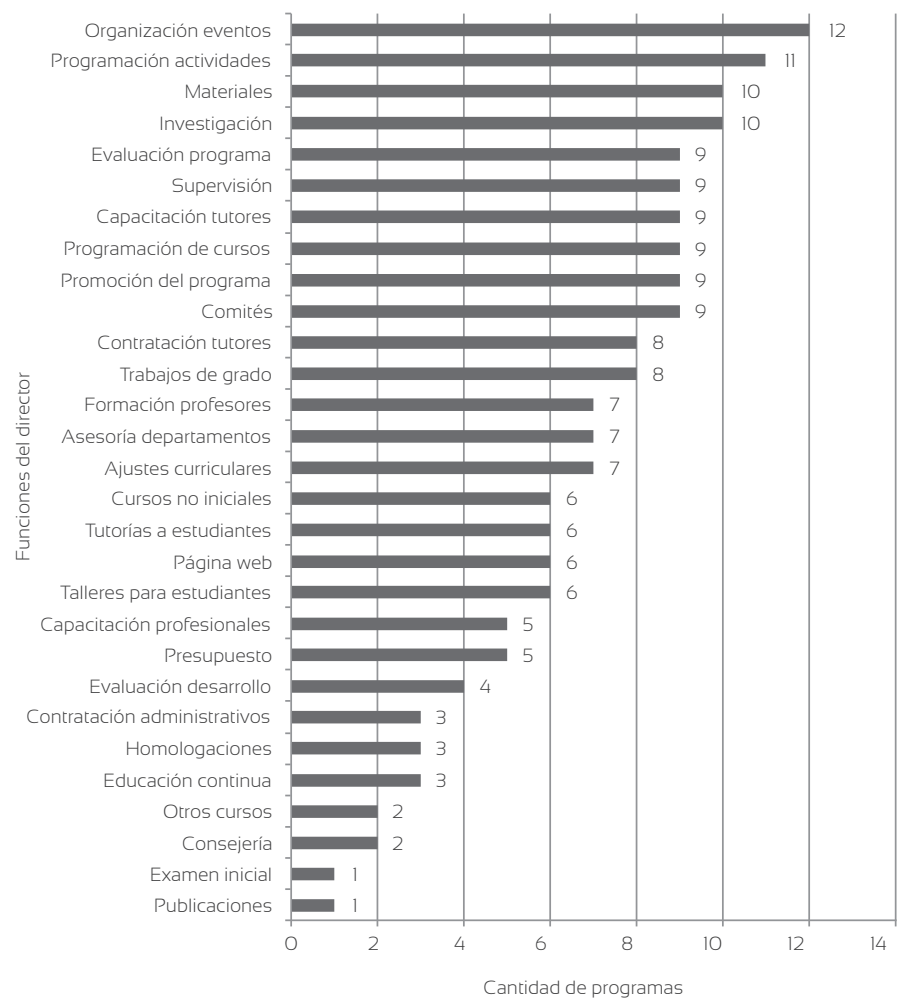

Figura 11

Funciones que desempeñan los directores

Fuente: elaboración propia.

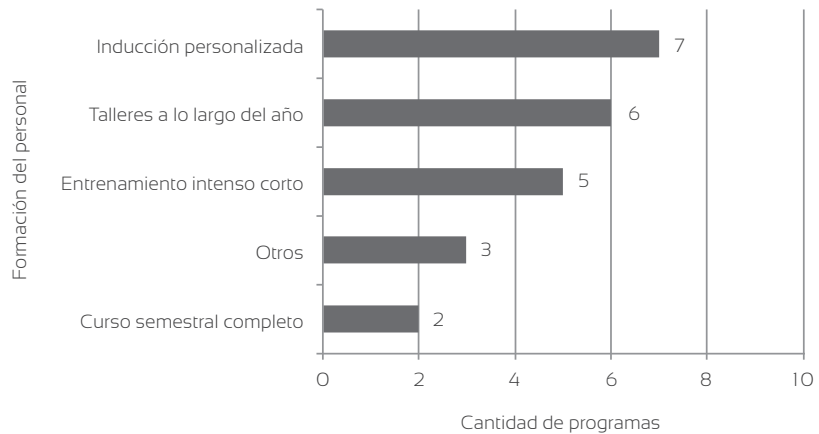

Figura 12

Formación del personal de los programas

Fuente: elaboración propia. 
Al aplicar las pruebas no paramétricas (Kruskal-Wallis y prueba de la mediana) con estadísticos de contraste entre algunas de las variables más importantes, no se evidencian diferencias significativas ( $p$ valor superior a 0,05) entre: el tipo de financiación de las instituciones y el número de servicios ofrecidos por los programas; el año de inicio de los programas y la cantidad de servicios ofrecidos; la cantidad de personal y la cantidad de servicios ofrecidos; el nivel educativo del director y la cantidad de funciones que debe desempeñar. Estos datos evidencian la complejidad a la que se enfrentan los centros y programas, pues independientemente del tiempo que tengan de creación o del perfil de su director, deben responder por una amplia gama de servicios, y los directores deben cumplir con funciones muy variadas, que trascienden las tareas propias de los programas y se extienden a funciones de docencia, investigación y extensión.

\section{Discusión y conclusiones}

Los datos presentados evidencian una variedad de programas muy jóvenes en su conformación. De la encuesta se puede deducir que para algunas universidades latinoamericanas la lectura y la escritura comienzan a estar en sus agendas programáticas, teniendo en cuenta el respaldo conferido a estas iniciativas. En el análisis de la encuesta se evidencia que este es un tema con un creciente interés por parte de instituciones de esta región. Cuando se analizan las razones para crear estos programas, prima la necesidad de mejorar la escritura, con las diferentes denominaciones que se le dan. Esto implica un reconocimiento de la escritura como un problema en la universidad (Peña, 2008), por tanto, al parecer la motivación que prima es remediar el problema a través de las estrategias y prácticas implementadas en estos programas.

Este enfoque fue el que los centros y programas de escritura tuvieron al inicio cuando se crearon entre las décadas de 1940 y 1970 (Russell, 2002), pero dista mucho de su quehacer actual, orientado hacia la escritura como proceso social (Roozen, 2015). Es probable que en Latinoamérica, al estar la gran mayoría de programas en una fase inicial, se esté considerando a los centros y programas de escritura como una estrategia que puede contribuir al mejoramiento de la prácticas de escritura (y lectura en varios casos), sin dejar de lado el trabajo que adelantan los cursos iniciales de lectura y escritura académica. Es necesario esperar varios años para conocer la tendencia que seguirán estos programas en la conformación de su propia identidad.

El desarrollo de un centro o programa de escritura requiere de una concepción amplia de la alfabetización académica como una herramienta de desarrollo del pensamiento y el conocimiento, como una acción social 
que conforma comunidades discursivas académicas y profesionales (Cisneros, Jiménez y Rojas, 2010). No obstante, la indagación muestra que este es el principal obstáculo cuando se inicia un programa de escritura en Latinoamérica, amén de los obstáculos de orden presupuestal y locativos. Este obstáculo puede verse como una oportunidad para los nacientes programas para proponer estrategias que fomenten la cultura académica en todas las instancias, desde estudiantes hasta directivos, y darse en la tarea de demostrar su importancia para el desarrollo de la calidad académica en sus instituciones. Se requiere, por supuesto, que en primer lugar al interior del programa exista claridad sobre estas concepciones, como lo sugieren la mayoría de estrategias implementadas para superar estos obstáculos por parte de los programas encuestados.

Para la creación de los programas o centros de escritura la evidencia señala que buscar ayuda con programas de mayor trayectoria es una estrategia poco empleada por la mayoría. Aunque se consulte información sobre otros programas, al parecer no existe la cultura de colaboración entre ellos, o al menos la intención de solicitarla. En cuanto a la fundamentación teórica y conceptual de los programas, no es del todo clara, teniendo en cuenta la escasa disponibilidad de fuentes bibliográficas para la mayoría de programas. Los textos en los cuales se fundamenta el proyecto de creación y posterior desarrollo de un centro o programa de escritura son, en su inmensa mayoría, textos generales sobre lectura y escritura. Frente a una pregunta muy específica sobre los textos especializados en centros o programas de escritura, las respuestas obtenidas iban desde manuales de escritura hasta diccionarios de español, pasando por libros de investigación científica o textos disciplinares. Lo anterior evidencia un desconocimiento de la literatura especializada sobre estos centros y programas, tanto por las referencias mencionadas como por la ausencia de respuesta de un número importante de programas al respecto.

Este desconocimiento sobre literatura especializada sobre centros y programas de escritura supone un reto significativo, pues es necesario contar con acceso a textos sobre el diseño, evaluación, investigación y administración de estos programas. Son muy pocas las universidades que cuentan con suscripciones a revistas o libros especializados de estos temas, aunque esto podría ser consecuencia del desconocimiento de quienes lideran dichos programas o a la falta de apoyo para adquirirlos por parte de las instituciones. El desarrollo de un programa de escritura no puede ir de espaldas a los avances teóricos existentes y es necesario implementar acciones colectivas para que el desconocimiento de dichos avances deje de ser un obstáculo y se pueda empezar a producir conocimiento derivado de las prácticas de los programas. Aunque los textos generales sobre alfabetización académica y escritura en la universidad son necesarios para entender y aplicar las prácticas de un centro o programa 
de escritura, los textos especializados pueden suplir el marcado desconocimiento sobre estos que evidencia la encuesta. Como lo plantea Lerner (2010):

Aunque formar relaciones estrechas con otros directores de centros de escritura es esencial para el éxito, comprender las relaciones de uno con los que han venido antes brinda una fuerte medida de continuidad y conexión e indica qué trabajo necesita hacerse. (p. 4) [Traducción propia].

Ahora bien, se observa que los programas incorporan prácticas que tienen que ver, en su mayoría, con el acompañamiento individual, bien sea a través de tutorías que buscan desarrollar habilidades de escritura, o bien de trabajo colaborativo entre docentes de lengua y docentes de las disciplinas. Es probable que el mayor número de centros de escritura versus el número de programas, tenga relación a que se proponen como estrategia de trabajo individual frente al trabajo grupal de los cursos. Otra razón por la que hay menos programas puede ser debido a que los cursos de lectura y escritura académica en su gran mayoría son ofrecidos desde departamentos de lengua y no desde programas específicos.

La gestión administrativa de estos programas plantea un desafío especial en cuanto a diversos aspectos. En primer lugar, en Latinoamérica no hay una formación específica para ser director de un programa de escritura y cumplir con una serie de funciones para las cuales se requiere una capacitación integral en tareas administrativas. Al igual que en otros contextos, estos directores provienen de formaciones variadas, con la diferencia de que en nuestros países no poseen experiencia en el trabajo al interior de uno de estos programas. Este aspecto apunta a la necesidad de generar capacitación específica en administración de programas de escritura en nuestra región y comenzar a formar futuros administradores o directores.

Un segundo aspecto de la gestión administrativa tiene que ver con la planeación. Lowe (2010) señala: "si fallas al planear, planeas fallar" (p. 71) para plantear las áreas de planeación estratégica que deben tener en cuenta los directores de centros de escritura. Los resultados de la encuesta muestran que los directores desempeñan en promedio 12 actividades diferentes, y algunos de ellos no cuentan con dedicación exclusiva para tales funciones. Esto, para los directores, puede significar una sobrecarga de trabajo o, como lo expresó Ede (1989), "el trabajo que hacemos es demandante y subvalorado, pero nunca consideraríamos cambiar de trabajo" (p. 3). La planeación estratégica requiere principios y valores (Adler-Kassner, 2013), pues "las estrategias sin ideales son una amenaza, pero los ideales sin estrategias son un desastre" (p. 396). De allí que las preguntas que plantea esta autora cobran validez para establecer los principios que se tengan al dirigir un 
programa de escritura: ¿Qué tipo de administrador de programa de escritura quieres ser? (En relación con el trabajo colaborativo o independiente); ¿qué tipo de alianzas quieres construir?, y ¿qué tipo de compromiso estás dispuesto a hacer? Estos cuestionamientos, y muchos más, son muy necesarios en el trabajo de un director de programa de escritura.

El tercer aspecto de la gestión administrativa que plantea desafíos es la evaluación. Medir el avance e impacto de un programa a partir de unos indicadores claros parece ser una premisa a seguir por casi la mitad de los centros de escritura aunque, en su gran mayoría, no cuentan con un modelo de gestión y evaluación. Si bien la mayoría ha adquirido estas habilidades y conocimientos por su experiencia en estos programas, es necesario fortalecer la administración de programas de escritura y hacerlo un campo de investigación (Molina-Natera, 2016). Por el lado de las instituciones, la falta de solicitud de evaluación de un programa, como le sucede a la mitad de ellos, puede indicar un escaso reconocimiento del aporte que puede hacer el programa a la gestión de la calidad académica. Les corresponde a los directores definir la misión de su programa para revisar periódicamente (al menos una vez al año) si se está siguiendo la senda definida por dicha misión para lograr la visión que se tenga del centro o programa. En el sentido planteado por White, Elliot y Peckham (2015), la evaluación de un programa de escritura es una investigación y este enfoque podría plantear desafíos más constructivos para los directores de estos programas.

Para los directores de centros y programas de escritura esta labor puede ser agobiante a veces, pero llena de satisfacciones. Los resultados de la encuesta evidencian que en todos los programas se trabaja fuerte para lograr sus objetivos, con los recursos con los que cada institución cuenta. En su reciente libro Caswell, McKinney y Jackson (2016) plantean tres tipos de trabajo por parte de un director: la labor emocional, la labor cotidiana y la labor disciplinar (o intelectual, en Geller y Denny, 2013). Los datos nos muestran que es necesario apoyar y reconocer estos tres tipos de trabajo de los directores y de los programas mismos. Aunque en la mayoría de casos se trata de posiciones nuevas para la institución, su desarrollo va de la mano con el desarrollo del programa mismo y, en un futuro próximo, con el desarrollo de la comunidad de centros y programas de escritura de nuestra región. 


\section{Referencias}

Adler-Kassner, L. (2013). What is principle? En R. Malenczyk (ed.), A Rhetoric for Writing Program Administrators (pp. 394-406). Anderson, SC: Parlor Press.

Backhoff, E., Velasco, V. y Peón, M. (2013). Evaluación de la competencia de expresión escrita argumentativa de estudiantes universitarios. Revista de la Educación Superior, XLII [Revista electrónica] (3), 9-39. Recuperado de: http://www.scielo.org.mx/scielo.php?script=sci_arttext\&pid=S0185-27602013000300001\&lng=es\&tlng=es [Fecha de consulta: 10 de mayo de 2016].

Banks, M. (2010). Los datos visuales en Investigación Cualitativa. Madrid: Ediciones Morata.

Bardin, L. (1996). Análisis de contenido. Madrid: Ediciones Akal S.A.

Carlino, P. (2005). Escribir, leer y aprender en la universidad. Una introducción a la alfabetización académica. Buenos Aires: Fondo de Cultura Económica.

Caswell, N., McKinney, J.G. y Jackson, R. (2016). The Working Lives of New Writing Centers Directors. Boulder, CO: Utah University Press.

Cisneros, M., Jiménez, H. y Rojas, G. (2010). Alfabetización académica y profesional como directrices de la acción formativa en la educación superior. En G. Parodi (ed.), Alfabetización académica y profesional en el siglo xxı: Leer y escribir desde las disciplinas (pp. 291-315). Santiago de Chile: Ariel.

Ede, L. (1989) Writing as a Social Process: A Theoretical Foundation for Writing Centers? Writing Center Journal, 9(2) 3-13.

García-Córdoba, F. (2002). El cuestionario: recomendaciones metodológicas para el diseño de cuestionarios. México: Limusa.

Geller, A.E. y Denny, H. (2013). Of Ladybugs, Low Status, and Loving the Job: Writing Center Professionals Navigating Their Careers. Writing Center Journal, 33(1), 96-129.

Gillespie, P. y Lerner, N. (2004). The Allyn and Bacon Guide to Peer Tutoring. 2a. ed. Nueva York: Pearson.

Gladstein, J. y Fralix, B. (2014). National Census of Writing. EE. UU. Recuperado de consulta: 15 de octubre de 2016].

González, B.Y. (2010). Strategies, Policies, and Research on Reading and Writing in Colombian Universities. En Ch. Bazerman et al. (eds.), Traditions on Writing Research. (pp. 122-132). New Jersey: Lawrence Erlbaum, New York: Routledge.

Harris, M. (1988). The Concept of a Writing Center. Urbana: The National Council of Teachers of English. [En línea]. Recuperado de: http://writingcenters.org/resources/starting-a-writing-cente/writing-center-concept/ 
International Writing Centers Association (IWCA) (2010). The Writing Centers Research Project. EE. UU. Recuperado de consulta: 15 de octubre de 2016].

Lerner, N. (2010). Time Warp: Historical Representations of Writing Centers Directors. En C. Murphy y B. Stay (eds.), The Writing Center Director's Resource Book (pp. 3-11). Mahwah, NJ: Routledge.

López-Noguero, F. (2002). El análisis de contenido como método de investigación. Revista de Educación XXI, 4, 167-179.

Lowe, K. (2010). "If You Fail to Plan, You Plan to Fail": Strategic Planning and Management for Writing Center Directors. En C. Murphy y B. Stay (eds.), The Writing Center Director Resource Book (pp.71-78). Mahwah, NJ: Routledge.

McLeod, S. (2007). Writing Program Administration. West Lafayette: Parlor Press. The WAC Clearinghouse. Recuperado de http://wac.colostate. edu/books/mcleod_wpa/

McMillan, J. y Schumacher, S. (2005). Investigación educativa. 5a. ed. Madrid: Pearson.

Molina-Natera, V. (2014). Centros de escritura: una mirada retrospectiva para entender el presente y futuro de estos programas en el contexto latinoamericano. Legenda, 18 (18), 9-33.

Molina-Natera, V. (ed.) (2015). Panorama de los Centros y Programas de Escritura de Latinoamérica. Cali, Colombia: Sello Editorial Javeriano.

Molina-Natera, V. (2016). Los centros de escritura en Latinoamérica: consideraciones para su diseño e implementación. En G. Bañales, M. Castelló y N. Vega (eds.), Enseñar a leer y escribir en la educación superior. Propuestas educativas basadas en la investigación (pp. 341362). México: Fundación SM.

Moyano, E. y Natale, L. (2012). Teaching Academic Literacy Across the University Curriculum as Institutional Policy: The Case of the Universidad Nacional de General Sarmiento (Argentina). En Ch. Thaiss et al. (eds.), Writing Programs Worldwide: Profiles of Academic Writing in Many Places (pp. 23-34). Anderson, Carolina del Sur: Parlor Press.

Olave, G., Rojas, I. y Cisneros, M. (2013). Deserción universitaria y alfabetización académica. Educación y Educadores, 16(3), 455-471.

Páramo, P. y Arango, M. (2008). Cuestionarios. En P. Páramo (comp.), La investigación en ciencias sociales. Técnicas de recolección de información (pp. 55-84). 2a. ed. Bogotá: Universidad Piloto de Colombia.

Peña, L.B. (2008). La competencia oral y escrita en la educación superior. Documento inédito del Comité Consultivo para la Definición de Estándares y Evaluación de Competencias Básicas en la Educación Superior. Bogotá: Ministerio de Educación Nacional. Recuperado de http://www.mineducacion.gov.co/1621/articles-189357_archivo_pdf_ comunicacion.pdf 
Pérez, M. y Rincón, G. (coords.) (2013). ¿Para qué se lee y se escribe en la universidad colombiana? Un aporte a la consolidación de la cultura académica del país. Bogotá: Pontificia Universidad Javeriana, Colciencias.

Rapley, T. (2014). Los análisis de la conversación, del discurso y de documentos en Investigación Cualitativa. Madrid: Ediciones Morata.

Roozen, K. (2015). Writing is a social and rhetorical activity. En L. Adler-Kassner y E. Wardle (eds.), Naming what we know. Threshold Concepts of Writing Studies (pp. 17-19). Boulder, CO: Utah State University Press.

Russell, D. (2002). Writing in the academic disciplines: A curricular history. 2a. ed. Carbondale and Edwardsville: Southern Illinois University Press.

Santos, D. (2016). La investigación acción participativa en la educación superior: el caso del programa LEA en la Universidad Nacional (UN) de Colombia. En G. Bañales, M. Castelló y N. Vega (eds.), Enseñar a leer y escribir en la educación superior. Propuestas educativas basadas en la investigación (pp. 315-337). México: Fundación SM.

White, E., Elliot, N. y Peckham, I. (2015). Very Like a Whale. The Assessment of Writing Programs. Boulder, CO: Utah University Press. 


\section{Anexo}

\section{Instituciones participantes}

\begin{tabular}{lc}
\hline \multicolumn{1}{c}{ Nombre de la institución } & Miembro de la RLCPE \\
\hline Tecnológico de Monterrey & Sí \\
Universidad de los Andes & Sí \\
Universidad Santiago de Cali & Sí \\
Fundación Universidad Internacional del Trópico & Sí \\
Centro de Investigación y Docencia Económicas & Sí \\
Universidad Autónoma Metropolitana & Sí \\
Universidad del Norte & Sí \\
Universidad Autónoma de Colombia & Sí \\
Instituto Tecnológico Autónomo de México & Sí \\
ICEsı & Sí \\
Universidad de Las Américas de Puebla & Sí \\
Universidad Santo Tomás & Sí \\
Universidad de Buenos Aires-Ciencias Sociales & No \\
Colegio Mayor de Cundinamarca & Sí \\
Pontificia Universidad Católica de Chile & Sí \\
Universidad de Chile & No \\
Universidad Autónoma de Occidente & Sí \\
Universidad del Cauca & Sí \\
Universidad Católica de Uruguay & Sí \\
Pontificia Universidad Javeriana Cali & Sí \\
Univerificia Universidad Católica Madre y Maestra & Sí \\
\hline Universidad del Turabo & Sucional de General Sarmiento \\
\hline
\end{tabular}

Fuente: elaboración propia. 